\title{
3D Surface Reconstruction Using Occluding Contours
}

\author{
Edmond Boyer and Marie Odile Berger \\ CRIN-CNRs / InRIA Lorraine \\ Campus scientifique, BP 239 \\ 54506 Vandoeuvre les Nancy Cedex, France \\ e-mail: boyere@loria.fr, berger@loria.fr
}

\begin{abstract}
This paper addresses the problem of $3 \mathrm{D}$ surface reconstruction using image sequences. It has been shown that shape recovery from three or more occluding contours of the surface is possible given a known camera motion. Several algorithms, which have been recently proposed, allow such a reconstruction under the assumption of a linear camera motion. A new approach is presented which deals with the reconstruction problem directly from a discrete point of view. First, a theoretical study of the epipolar correspondence between occluding contours is achieved. A correct depth formulation is then derived from a local approximation of the surface up to order two. This allows the local shape to be estimated, given three consecutive contours, without any constraints on the camera motion. Experimental results are presented for both synthetic and real data.
\end{abstract}

A short version of this article appears in the Proceedings of 6th International Conference on Computer Analysis of Images and Patterns, Prague, september 1995 and a long version will appear in the International Journal of Computer Vision (accepted for publication).

\section{Introduction}

Recovering three-dimensional shapes from visual data is an important intermediate goal of many vision systems used in robotics, surveillance, guidance and modelling. In such applications, surface reconstruction is often used as an intermediate step in the more important task of 3D representation and recognition.

For non-polyhedral objects, rich and robust information on the shape are provided by the occluding contours. In fact, if some strong a priori knowledge on the object is available such as parametric descriptions, then a single view allows shape recovery [Pon 89, Zer 93], object pose estimation and object recognition [Kri 90, Gla 92, For 92]. Otherwise, for any smooth object, a sequence of occluding contours must be considered to recover the shape.

The problem of reconstructing surfaces from occluding contours was first considered by Koenderink [Koe 84], who attempted to infer 3D curvature properties from occluding contours. Later, Giblin and Weiss [Gib 87] proved that surface reconstruction can be performed for planar motion 
under the assumption of orthographic projection. The problem of reconstructing under perspective projection for general known motions has been tackled by Cipolla and Blake [Cip 90], and Vaillant [Vai 92]. Both approaches lead to depth computation under the assumption of continuous observations of the occluding contours and are based on differential analysis. Unfortunately, the use of differential computations with discrete observations requires numerical approximations and produces numerical instabilities. With the aim of further constraining the problem, the occluding contour is supposed to be locally part of a circle. In addition, the plane containing the circle is supposed to be known: in [Cip 90], the epipolar plane is used whereas Vaillant [Vai 92] used the radial plane.

While keeping the circle model, Szeliski [Sze 93] proposed to improve the reconstruction process by computing the epipolar curves on the whole surface together with an estimate of uncertainty. The use of estimation theory leads to a more robust shape recovery using a linear smoother but the basic reconstruction method remains unchanged.

The approaches described above only allow a $3 \mathrm{D}$ depth map of points or curves belonging to the surface to be obtained. In a recent work, Zhao [Zha 94] attempts to recover the global 3D surface description from the occluding contours in a single stage by use of B-spline patches. This approach introduces a direct regularisation of the reconstructed surface, but it requires a complete a priori parametrisation of the surface which is usually not available. Therefore it is preferable to first recover a more accurate 3D depth map before computing a 3D surface description.

The task considered in this paper is to deal with the reconstruction problem directly from a discrete point of view. We first address the problem of the mapping between successive occluding contours and we give new results in the case of the epipolar correspondence. Then, a correct depth formulation is given when the occluding contours are observed at discrete times. The main interest of such a formulation is to avoid reconstruction constraints (unlike [Cip 90, Vai 92, Sze 93, Jos 95] no reconstruction plane is locally imposed), so that non-linear motion can be easily taken into account. Moreover, it gives a general solution to the reconstruction problem which is always defined except when the camera motion is in the viewing direction. Our approach is based on a local approximation of the surface up to second order, allowing a linear estimation of depth to be derived, given any set of three occluding contours.

This paper is organised as follows: the epipolar correspondence induced between two discrete observations is studied in section 2. In section 3, the depth formulation is established, enabling the discrete observations of occluding contours to be handled. In Section 4, our approach is compared to previous methods. The algorithm performances are studied in section 5 and significant reconstruction results on real data are shown in section 6 , before concluding. 


\section{Viewing geometry}

Let $P$ be a point on a smooth curved surface ${ }^{1} \mathcal{S}$. We assume that the imaging system is based on the pinhole model (i.e. perspective projection). Therefore, the vector position $r$ of $P$ can be written

$$
r=C+\lambda T,
$$

where $C$ is the camera centre position, $T$ the unit viewing direction and $\lambda$ the depth of the point $P$ along the viewing direction. For a given camera position there is a locus of points on the surface $\mathcal{S}$ where the normal $N$ is perpendicular to the viewing direction. This set of points is called the rim (also known as the limb) and its projection onto the image plane is called the occluding contour (also known as the extremal boundary or the profile), as shown in figure 1.

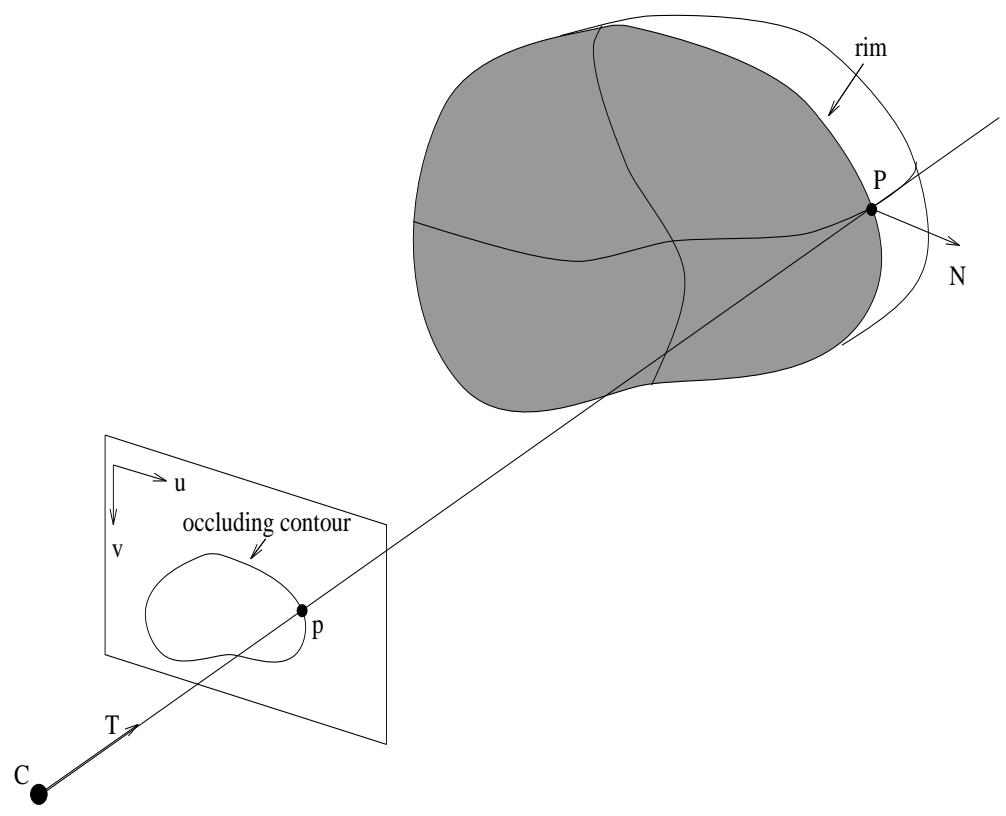

Figure 1: Rim and occluding contour under perspective projection.

As the camera moves around $\mathcal{S}$ the occluding contours sweep a surface in the space of parameters $(u, v, t)$ which is called the spatio-temporal surface [Fau 93, Gib 95].

Since the camera centre position is a function of time $t$, this surface can be parameterised by $(s, t)$, where the parameter $s$ describes the position on the occluding contours and the parameter $t$ corresponds to time. However, such a parametrisation is not uniquely defined [Cip 90]: curves of constant $t$ are the occluding contours but curves of constant $s$ have no physical interpretation. Until now, the most generally accepted parametrisation of the spatio-temporal surface is the epipolar parametrisation which yields a mapping between successive occluding contours called the epipolar correspondence. The advantage of this parametrisation is that it leads to a local parametrisation of the surface $\mathcal{S}$ which can always be used except when the occluding contour is singular or when

\footnotetext{
${ }^{1}$ We suppose that the surface is at least $C^{2}$ and that it is not locally a plane.
} 
the camera motion is in the plane tangent to the surface [Gib 95]. Furthermore, the epipolar correspondence between points on successive occluding contours constrains the reconstruction problem which becomes a linear estimation, as shall be seen later.

\section{$2.1 \quad$ Epipolar correspondence}

The epipolar parametrisation is derived from the epipolar geometry in stereo-vision. It leads to a planar correspondence between two successive occluding contours as shown in figure 2. Hence, two points $p_{1}$ and $p_{2}$ on two successive occluding contours are epipolar correspondents if they both belong to the epipolar plane defined by the two camera centres and one of the points. Such correspondences between points on the spatio-temporal surface generally induce correspondences between points on the surface $\mathcal{S}$. For a continuous motion of the camera, curves of constant $s$ on the spatio-temporal surface induce curves on the surface $\mathcal{S}$. These curves are called epipolar curves. In this section we focus on the local behaviour of the epipolar correspondence between two occluding contours of $\mathcal{S}$.

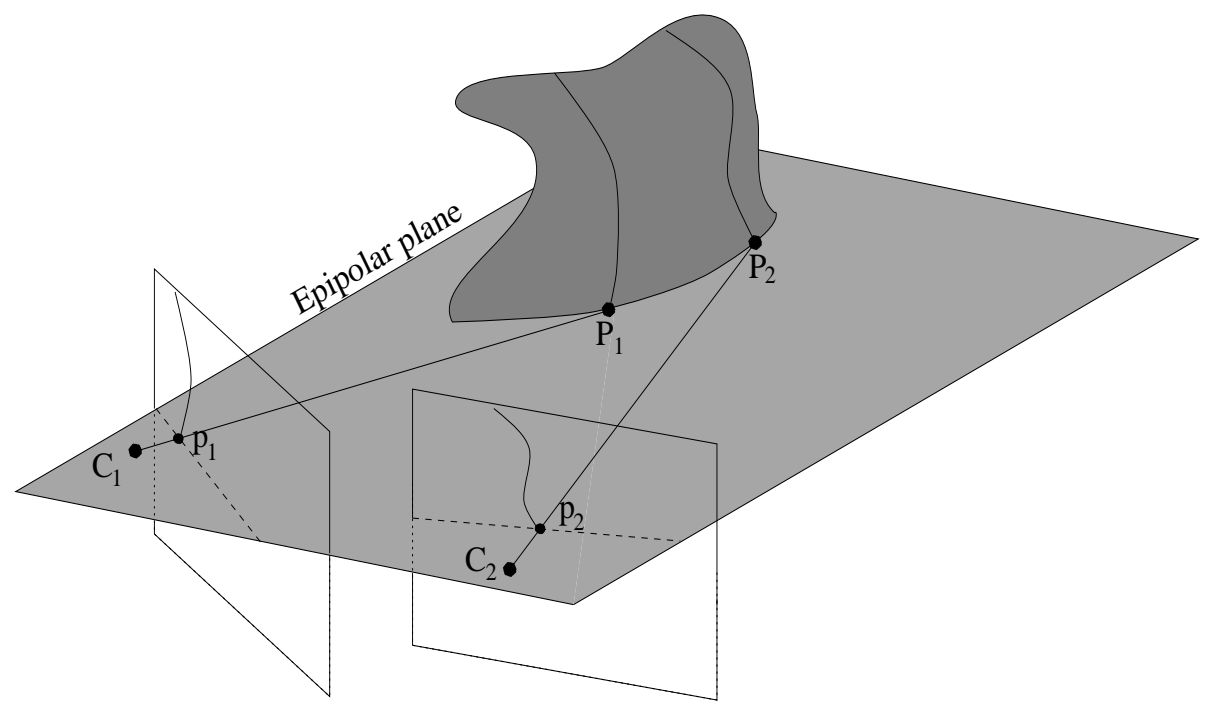

Figure 2: Two epipolar correspondents $p_{1}$ and $p_{2}$.

Consider two occluding contours $\mathcal{O}_{1}$ and $\mathcal{O}_{2}$ of $\mathcal{S}$ for two successive camera centre positions $C_{1}$ and $C_{2}$. We denote by $T_{1}\left(s_{1}\right)$ the viewing direction for points $p_{1}$ on $\mathcal{O}_{1}$ where the parameter $s_{1}$ describes the position on $\mathcal{O}_{1}$, and equivalently by $T_{2}\left(s_{2}\right)$ the viewing direction for points $p_{2}$ on $\mathcal{O}_{2}$ where the parameter $s_{2}$ describes the position on $\mathcal{O}_{2}$. The epipolar correspondence leads to the following matching constraint between two image points $p_{1}$ and $p_{2}$ on $\mathcal{O}_{1}$ and $\mathcal{O}_{2}$ :

$$
F\left(s_{1}, s_{2}\right)_{\mid\left(p_{1}, p_{2}\right)}=\left(T_{1}\left(s_{1}\right) \wedge T_{2}\left(s_{2}\right)\right) \cdot\left(C_{1}-C_{2}\right)=0
$$

In the general case, epipolar correspondents $p_{1}$ and $p_{2}$ are image projections of two different 
points $P_{1}$ and $P_{2}$ on $\mathcal{S}$. This non-stationarity property of occluding contours can be used to discriminate such contours from others [Vai 92]. However, there is a circumstance where the property is not verified. This occurs when the camera motion $\left(C_{1}-C_{2}\right)$ is in the tangent plane of $\mathcal{S}$ at $P_{1}$ or $P_{2}$. In this situation, points $p_{1}$ and $p_{2}$ are image projections of the same fixed point $P$ $\left(P=P_{1}=P_{2}\right)$, and rims of $\mathcal{S}$ for camera positions $C_{1}$ and $C_{2}$ intersect at $P$. We call such a point $P$ a multiple point of the sequence considered.

Definition $A$ multiple point of an image sequence of $\mathcal{S}$ is a point $P$ where two or more consecutives rims of the sequence intersect.

Consequently, if $P$ is a multiple point of an image sequences of $\mathcal{S}$ then the epipolar plane at $P$ is tangent to $\mathcal{S}$. In addition, if the $n$ camera centre positions of the image sequences are aligned, then $P$ is of multiplicity $n$.

Remark In the literature, the locus of rim points where the epipolar plane is tangent to the surface is called the frontier [Gib 95, Cip 95]. For a linear camera motion, the frontier, if it exists, is restricted to isolated points. Thus, multiple points represent the frontier for linear camera motions going through successive camera centre positions of the sequence. Since these rim points are fixed features on the surface, they can be used to derive constraint on the camera motion [Rie 86, Por 91, Cip 95, Jos 95].

Equation (2) relates parameters $s_{1}$ and $s_{2}$ of two epipolar correspondents. Now consider the epipolar map:

$$
f: \mathcal{O}_{1} \rightarrow \mathcal{O}_{2}
$$

which associates with every point $p_{1} \in \mathcal{O}_{1}$ its epipolar correspondent $p_{2} \in \mathcal{O}_{2}$ such that $F\left(s_{1}, s_{2}\right)_{\mid p_{1}, p_{2}}=$ 0 . Assuming that the occluding contours are $\operatorname{smooth}^{2}$, we have:

Proposition 1 The epipolar map $f$ is a local diffeomorphism at $p_{1}$ if the point $P_{1}$ on $\mathcal{S}$, with image projection $p_{1}$, is not a multiple point of the sequence considered.

Proof. This proposition is a direct consequence of the implicit function theorem [Bru 84] applied to the function $F$. We denote by $s_{p_{1}}$ and $s_{p_{2}}$ the parameters of two epipolar correspondents $p_{1}$ and $p_{2}$ on $\mathcal{O}_{1}$ and $\mathcal{O}_{2}$, therefore $F\left(s_{p_{1}}, s_{p_{2}}\right)=0$. If the occluding contours are smooth, then $T_{1}\left(s_{1}\right)$, $T_{2}\left(s_{2}\right)$ are smooth with respect to $s_{1}$ and $s_{2}$ and the function $F\left(s_{1}, s_{2}\right)$ is smooth. By the implicit function theorem, there exists a continuous map $f: s_{1} \mapsto s_{2}$ which is smooth in a neighbourhood of $s_{p_{1}}$ provided $\frac{\partial F}{\partial s_{2}}\left(s_{p_{1}}, s_{p_{2}}\right) \neq 0$. Likewise $s_{1}$ is a smooth function of $s_{2}$ in a neighbourhood of $s_{p_{2}}$ provided $\frac{\partial F}{\partial s_{1}}\left(s_{p_{1}}, s_{p_{2}}\right) \neq 0$. Rewriting (2) and taking the derivatives with respect to $s_{1}$ and $s_{2}$, we

\footnotetext{
${ }^{2}$ Under the hypothesis we made on $\mathcal{S}$ (i.e. smooth and not locally a plane) and for a generic viewpoint, occluding contours are locally smooth except at a cusp or a T-junction [Koe 86].
} 
obtain:

$$
\left\{\begin{array}{l}
\frac{\partial F}{\partial s_{2}}\left(s_{p_{1}}, s_{p_{2}}\right)=\left(\left(C_{1}-C_{2}\right) \wedge T_{1}\left(s_{p_{1}}\right)\right) \cdot \frac{\partial T_{2}}{\partial s_{2}}\left(s_{p_{2}}\right), \\
\frac{\partial F}{\partial s_{1}}\left(s_{p_{1}}, s_{p_{2}}\right)=\left(\left(C_{2}-C_{1}\right) \wedge T_{2}\left(s_{p_{2}}\right)\right) \cdot \frac{\partial T_{1}}{\partial s_{1}}\left(s_{p_{1}}\right) .
\end{array}\right.
$$

The epipolar plane at $p_{2}$ is spanned by $T_{2}\left(s_{p_{2}}\right)$ and $\left(C_{2}-C_{1}\right)$, or equivalently by $T_{1}\left(s_{p_{1}}\right)$ and $\left(C_{1}-C_{2}\right)$. Thus $\frac{\partial F}{\partial s_{2}}\left(s_{p_{1}}, s_{p_{2}}\right)=0$ if $\frac{\partial T_{2}}{\partial s_{2}}\left(s_{p_{2}}\right)$ belongs to the epipolar plane at $p_{2}$. Since $T_{2}\left(s_{p_{2}}\right)$ and $\frac{\partial T_{2}}{\partial s_{2}}\left(s_{p_{2}}\right)$ both belong to the tangent plane of $\mathcal{S}$ at $P_{2}$, this occurs at points $p_{1}$ and $p_{2}$ where the epipolar plane is tangent to the surface. In this case, $P_{2}$ is a multiple point and $P_{1}=P_{2}$. Likewise $\frac{\partial F}{\partial s_{1}}\left(s_{p_{1}}, s_{p_{2}}\right)=0$ if the epipolar plane is tangent to the surface and thus $P_{2}=P_{1}$. The map $f: s_{1} \mapsto s_{2}$ is therefore a local diffeomorphism at $s_{p_{1}}$ if the point $p_{1}$ is not the projection of a multiple point..

Proposition 1 says that given two epipolar correspondents $p_{1}$ and $p_{2}$, there exist neighbourhoods of $p_{1}$ and $p_{2}$ where the epipolar map is smooth and has a smooth inverse, provided that $p_{1}$ and $p_{2}$ are not projections of a multiple point. In fact, in the neighbourhood of the projection of a multiple point, the epipolar map yields two correspondents, as shown in figure 3. The epipolar

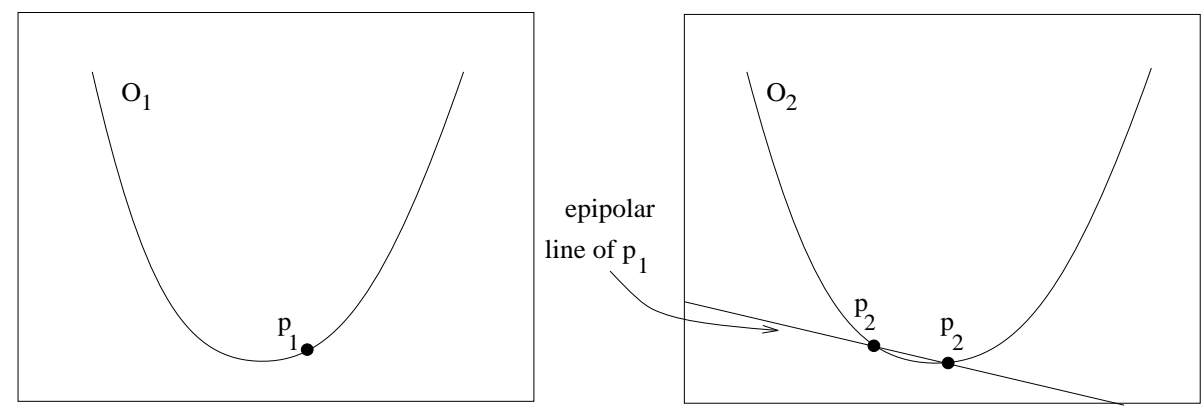

Figure 3: The epipolar correspondence is ambiguous in the proximity of a multiple point: $p_{1}$ has two close correspondents $p_{2}$.

correspondence is thus ambiguous in the proximity of a multiple point. The criterion one can use to resolve this ambiguity is that the image of $P_{1}, P_{2}$ and the multiple point must appear in the same order along occluding contours (this assumes that occluding contours have the same orientation). Note that this criterion is similar to the ordering constraint in stereo-vision.

\subsection{Recovering depth along the view line}

Our goal is to recover rims from their projections. First, we suppose that under a continuous motion of the camera a complete description of the spatio-temporal surface is available. Then, (1) can be derived according to time $t$ and by taking the scalar product with the normal $N$ to the surface, we obtain:

$$
\lambda=\frac{-\frac{\partial C}{\partial t} \cdot N}{\frac{\partial T}{\partial t} \cdot N}
$$


This is the depth formula for points on rims as defined in [Cip 90]. Assuming that we have a parametrisation of $\mathcal{S}$, we can therefore compute $\frac{\partial T}{\partial t}$ from the spatio-temporal surface and then recover the depth for points on the spatio-temporal surface where $\frac{\partial T}{\partial t} \cdot N \neq 0$. Unfortunately, only discrete information (occluding contours at discrete times $t_{i}, i \in[1 . . m]$ ) are available and (3) can not be applied directly. In fact, depth can be computed only by approximation. In this section, we discuss such an approximation.

Now consider two successive occluding contours at times $t_{1}$ and $t_{2}$. Let $P_{1}$ and $P_{2}$ be two points on the rims of $\mathcal{S}$ at $t_{1}$ and $t_{2}$. Using the notations of the previous section, we have:

$$
\begin{cases}r_{1}=C_{1}+\lambda_{1} T_{1} & \text { at } P_{1}, \\ r_{2}=C_{2}+\lambda_{2} T_{2} & \text { at } P_{2}\end{cases}
$$

Thus, denoting by $\Delta x$ the difference $\Delta x=x_{1}-x_{2}$, where $x$ is any of $r, C, \lambda$ and $T$, gives:

$$
\Delta r=\Delta C+\lambda_{1} \Delta T+\Delta \lambda T_{2}
$$

and by taking the scalar product with the normal $N_{2}$ to the surface at $P_{2}$, we obtain the following depth formula for point $P_{1}$ :

$$
\lambda_{1}=\frac{-\Delta C \cdot N_{2}}{\Delta T \cdot N_{2}}+\frac{\Delta r \cdot N_{2}}{\Delta T \cdot N_{2}} .
$$

If the image projections $p_{1}$ and $p_{2}$ of $P_{1}$ and $P_{2}$ are matched according to the epipolar correspondence, then the first term of the above expression corresponds to a triangulation formula. Indeed, $\frac{-\Delta C \cdot N_{2}}{\Delta T \cdot N_{2}}$ is the distance from the camera centre $C_{1}$ to the viewlines intersection (see figure 4). This value is therefore the depth of a virtual point with image projection $p_{1}$ and $p_{2}$. Hence, it can be computed from measurements in two images by using a stereo formula.

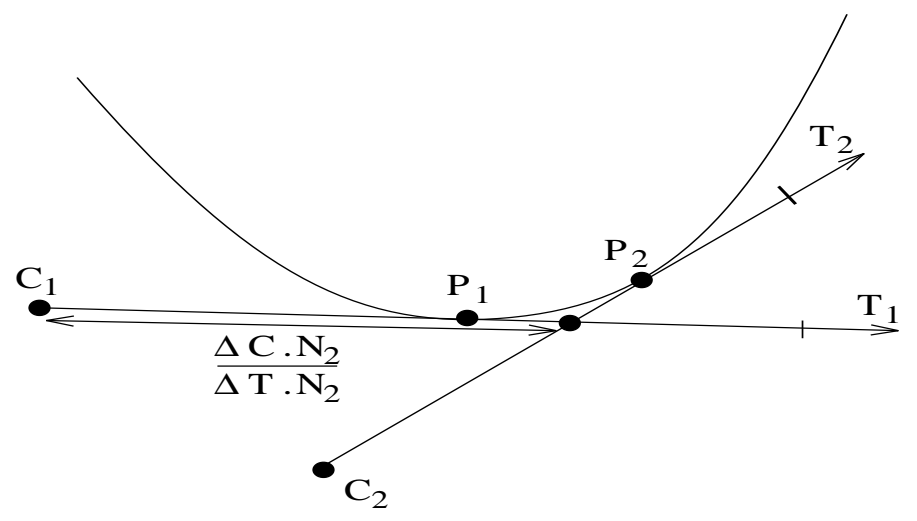

Figure 4: Intersection of tangents in the epipolar plane.

On the other hand, the second term of (4) depends on the distance $\Delta r$ between surface points $P_{1}$ and $P_{2}$. This value can not be computed, a priori, from measurements in two images. A first 
approach would consist in omitting this term in (4). We could then recover the depth of a surface point with only two images. But this approach leads to a stereo reconstruction and implicitly assumes that rims are not view dependent which is of course wrong. For a smooth surface which is not locally plane, $\Delta r . N_{2} \neq 0$ except at a multiple point. Therefore, the second term of (4) should not be omitted when computing depth.

The approach we have developed is based on a local surface model: a second order approximation. Such a model allows $\Delta r . N_{2}$ to be expressed as a function of local properties of $\mathcal{S}$. Hence, by using more than two images (three for a second order approximation), we can fit locally the surface model to the image measurements and estimate both depth and local properties. This idea is developed in the following section.

\section{Reconstruction}

We have seen in the previous section that more than two images are needed to estimate the local properties of the surface $\mathcal{S}$. We show here that by using three images, a local approximation up to order 2 of $\mathcal{S}$ can be computed. Moreover, if points between occluding contours of $\mathcal{S}$ are matched according to the epipolar correspondence, this approximation leads to a linear estimation of the position and the curvatures of $\mathcal{S}$ at a point $P$. Unlike previous methods [Cip 90, Vai 92, Sze 93, Jos 95] which use an a priori plane in order to estimate the epipolar curve, no assumption is made on the camera motion or the local surface shape. Instead, a local parametrisation of the surface $\mathcal{S}$ is used which in turn leads to a local surface approximation. We first present the local parametrisation $(x, y)$ that is used and the induced local approximation of the surface. We show then that such a parametrisation allows linear equations to be derived for both depth and normal curvature in the viewing direction.

\subsection{The osculating quadric}

Since $\mathcal{S}$ is a smooth surface, a neighbourhood of a point $P$ on $\mathcal{S}$ can be represented in the form $z=h(x, y)$, where $P$ is the origin of the coordinate frame and the $z$ axis is directed by the normal $N$ of $\mathcal{S}$ at $P$. Thus the $x y$ plane is the tangent plane to $\mathcal{S}$ at $P$. Moreover, $h$ is a differentiable function and by taking Taylor's expansion at $P$, we have:

$$
h(x, y)=z=\frac{1}{2}\left(h_{x x} x^{2}+2 h_{x y} x y+h_{y y} y^{2}\right)+R(x, y),
$$

where $R(x, y)$ satisfies:

$$
\lim _{(x, y) \rightarrow(0,0)} \frac{R(x, y)}{x^{2}+y^{2}}=0 .
$$


The quadratic form $\frac{1}{2}\left(h_{x x} x^{2}+2 h_{x y} x y+h_{y y} y^{2}\right)$ is known as the Hessian of $h$ at $(0,0)$ and corresponds to the second fundamental form of $\mathcal{S}$ at $P$. The quadratic surface $\mathcal{Q}$ defined by

$$
\mathcal{Q}=\left\{(x, y, z), z=\frac{1}{2}\left(h_{x x} x^{2}+2 h_{x y} x y+h_{y y} y^{2}\right)\right\}
$$

approximates $\mathcal{S}$ up to order 2. This surface is called the osculating quadric of $\mathcal{S}$ at $P$ and is uniquely defined.

We first assume that the $x$ and $y$ axes are oriented by $T$ and $r_{s} /\left|r_{s}\right|$ respectively, where $T$ is the viewing direction and $r_{s}$ the tangent to the rim of $\mathcal{S}$ at $P$. Since these directions are conjugate [Koe 84], the first and second fundamental forms ${ }^{3}$ of $\mathcal{S}$ at $P$, in the parametrisation $(x, y)$, are:

$$
I_{P}=\left[\begin{array}{cc}
1 & \cos \theta \\
\cos \theta & 1
\end{array}\right] \quad I I_{P}=\left[\begin{array}{cc}
k_{t} & 0 \\
0 & k_{s}
\end{array}\right]
$$

where $\theta$ is the angle between $T$ and $r_{s}, k_{t}$ is the normal curvature along the viewing direction $T$, and $k_{s}$ is the normal curvature of the rim at $P$. Note that $k_{t}$ is the normal curvature of the epipolar curve at $P$. Therefore, the osculating quadric of $\mathcal{S}$ at $P$ is defined, in the parametrisation $(x, y)$, by:

$$
\mathcal{Q}=\left\{(x, y, z), z=\frac{1}{2}\left(k_{t} x^{2}+k_{s} y^{2}\right)\right\}
$$

We denote by $\mathcal{E}_{-1}$ and $\mathcal{E}_{1}$ the epipolar planes at a point $P \in \mathcal{S}$ corresponding to three successive positions of the camera centre $C_{-1}, C$ and $C_{1}$, i.e., planes $\left(C, C_{-1}, P\right)$ and $\left(C, C_{1}, P\right)$ (see figure $5)$. The point $P$ is therefore a point belonging to the rim observed from $C$. For a general motion $\mathcal{E}_{-1}$ and $\mathcal{E}_{1}$ are different (they are identical for a linear motion). The intersection of one epipolar plane with the surface $\mathcal{S}$ is a curve. By abuse of language, we call these curves epipolar curves ${ }^{4}$ and we have then the following property:

Proposition 2 In any epipolar plane $\mathcal{E}$ at $P$, the epipolar curve is, up to the order 2 , the graph of the following function:

$$
z_{\mathcal{E}}=g(x)=\frac{1}{2} \frac{k_{t}}{\cos \beta_{\mathcal{E}}} x^{2},
$$

where the $x$ axis is directed by $\left.T\right|_{P}$, the $z_{\mathcal{E}}$ axis is such that $\left(x, z_{\mathcal{E}}\right)$ form an orthonormal basis of $\mathcal{E}$ and $\beta_{\mathcal{E}}$ is the angle between the normal $N$ to the surface at $P$ and the projection $N_{\mathcal{E}}$ of $N$ in the epipolar plane: $\cos \beta_{\mathcal{E}}=N . N_{\mathcal{E}}$.

\footnotetext{
${ }^{3}$ See $[\mathrm{dC} 76]$ for definitions.

${ }^{4} \mathrm{As}$ introduced in 2.1 , the epipolar curve is defined for a continuous camera motion. Thus, the curves under considerations are the epipolar curves for linear camera motions going through two successive camera centre positions.
} 
Proof. Near the point $P$, we have the following description of $\mathcal{S}$ :

$$
z=\frac{1}{2}\left(k_{t} x^{2}+k_{s} y^{2}\right)+R(x, y)
$$

with $\lim _{(x, y) \rightarrow(0,0)} R(x, y) /\left(x^{2}+y^{2}\right)=0$.

Let $\mathcal{E}$ be an epipolar plane at $P$ and let $\beta_{\mathcal{E}}$ be the angle between the normal $N$ to $\mathcal{S}$ at $P$ and its projection $N_{\mathcal{E}}$ in $\mathcal{E}$. The equation of this plane can be written as:

$$
z \sin \beta_{\mathcal{E}}=y \cos \beta_{\mathcal{E}} \sin \theta
$$

where $\theta$ is the angle between the $x$ axis and the $y$ axis (or equivalently between $T$ and $r_{s}$ at $P$ ). Now if the $z_{\mathcal{E}}$ axis is defined such that $\left(x, z_{\mathcal{E}}\right)$ forms an orthonormal basis of $\mathcal{E}$, we have:

$$
z=z_{\mathcal{E}} \cos \beta_{\mathcal{E}}
$$

The epipolar curve is the intersection of the plane $\mathcal{E}$ with the surface $\mathcal{S}$, therefore by substituting in (7) and neglecting third order and higher terms we obtain for points on these curves:

$$
z_{\mathcal{E}} \cos \beta_{\mathcal{E}}=\frac{1}{2}\left(k_{t} x^{2}+\frac{\sin ^{2} \beta_{\mathcal{E}}}{\sin ^{2} \theta} k_{s} z_{\mathcal{E}}^{2}\right)
$$

In general, the term $\frac{\sin ^{2} \beta_{\varepsilon}}{\sin ^{2} \theta} k_{s}$ in (8) can be considered as bounded since:

1. the case $\sin \theta=0$ happens only when $P$ is observed along an asymptotic direction which yields a cusp on the occluding contour.

2. The curvature $k_{s}$, which is linked to the curvature of the occluding contour, is finite in our context (the observed object is smooth).

Thus, solving $(8)$ for $\left(x, z_{\mathcal{E}}\right)$ close to $(0,0)$ yields:

$$
\begin{cases}z_{\mathcal{E}}=\frac{1}{2} \frac{k_{t}}{\cos \beta_{\mathcal{E}}} x^{2}+R^{\prime}(x), & \cos \beta_{\mathcal{E}} \neq 0 \\ z_{\mathcal{E}}=x=0, & \cos \beta_{\mathcal{E}}=0\end{cases}
$$

with: $\lim _{x \rightarrow 0} R^{\prime}(x) / x^{4}=0$. This shows that up to second order, the epipolar curve is represented by:

$$
z_{\mathcal{E}}=\frac{1}{2} \frac{k_{t}}{\cos \beta_{\mathcal{E}}} x^{2}, \quad \quad \cos \beta_{\mathcal{E}} \neq 0
$$

The case $\cos \beta_{\mathcal{E}}=0$ occurs when $P$ is a multiple point, the epipolar curve is then restricted to a single point $P$.

Remark The approximation given in proposition 2 verifies the Meusnier's theorem [dC 76] which says that the curvature at $P$ of the epipolar curve is $k=\frac{k_{t}}{\cos \beta_{\varepsilon}}$. However, it should be noticed that 
a local approximation of the epipolar curve based on a circle verifies also Meusnier's theorem. But such approximation implicitely implies that the surface is locally spherical which is less general than the osculating quadric model.

Note that in the above proposition, the $x$ axis is the one previously defined in the parametrisation $(x, y)$ and is thus independent of the epipolar plane.

Since $P$ is the origin of the $x$ axis, proposition 2 says that the epipolar curve depends on the position of $P$ in the epipolar plane (i.e., its depth) and on the normal curvature $k_{t}$ of $\mathcal{S}$ along the viewing direction. Our purpose is to recover the position of a rim point $P$ using three successive occluding contours of $\mathcal{S}$, therefore this can be done by estimating epipolar curves. In the general case there are two different epipolar planes $\mathcal{E}_{-1}$ and $\mathcal{E}_{1}$ for a point $P$ and three successive camera positions, thus there are also two epipolar curves. Since we can match, in the corresponding images, epipolar correspondents, we know two tangents to each epipolar curve (see figure 5). The following section shows how to compute epipolar curves given these tangents.

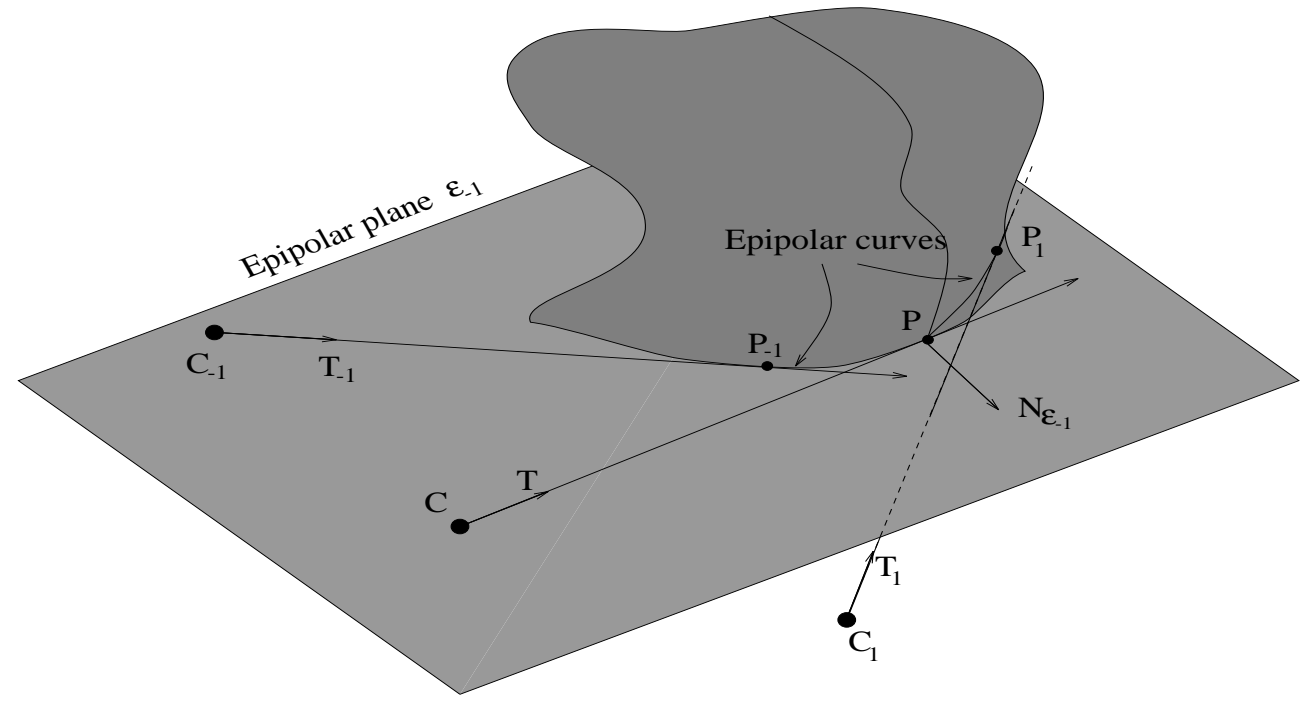

Figure 5: Epipolar curves.

\subsection{Estimation of the epipolar curves}

Our goal is to recover the depth $\lambda$ and the curvature $k_{t}$ at $P$. It has been shown that these values are related to the position and the curvature of the epipolar curves. Therefore, the problem is to estimate two of these curves given three tangents. The previous section showed that the epipolar curves are, up to order 2, parabolas of the epipolar planes. Moreover, although the epipolar curves are not in the same plane, by using proposition 2 , linear estimations of both $\lambda$ and $k_{t}$ can be derived.

Now consider the epipolar plane $\mathcal{E}_{1}$. We denote by $\beta_{\mathcal{E}_{1}}$ the angle between the normal $N$ at $P$ 
and its projection $N_{\mathcal{E}_{1}}$ in $\mathcal{E}_{1}$ :

$$
\cos \beta_{\mathcal{E}_{1}}=N . N_{\mathcal{E}_{1}} .
$$

By proposition 2, the epipolar curve with tangents $T$ and $T_{1}$ is defined by (up to order two):

$$
z_{\mathcal{E}_{1}}=\frac{1}{2} k_{\mathcal{E}_{1}} x^{2}, \quad k_{\mathcal{E}_{1}}=\frac{k_{t}}{\cos \beta_{\mathcal{E}_{1}}}
$$

where $\left(x, z_{\mathcal{E}_{1}}\right)$ form an orthogonal basis of $\mathcal{E}_{1}$. Let $x_{P_{1}}$ be the abscissa of $P_{1}$ as shown in figure 6 .

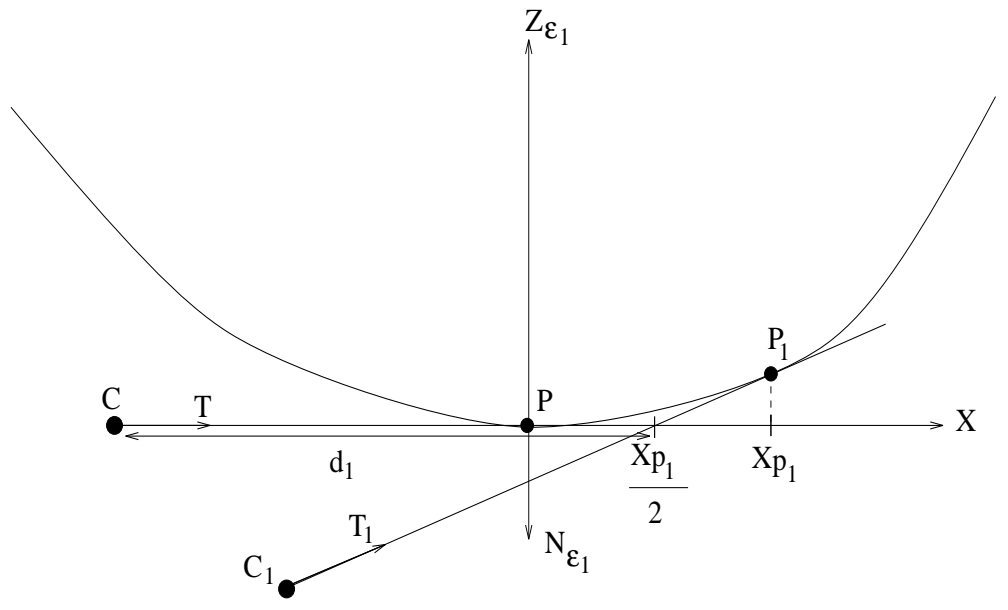

Figure 6: The epipolar plane $\mathcal{E}_{1}$.

Since $P_{1}$ belongs to the epipolar curve, the tangent to $\mathcal{S}$ at $P_{1}$ in the direction $T_{1}$ goes through the point $\left(\frac{x_{P_{1}}}{2}, 0\right)$ and it follows that:

$$
\left\{\begin{array}{l}
T_{1} \cdot N_{\mathcal{E}_{1}}=\frac{k_{\mathcal{E}_{1}} x_{P_{1}}^{2} / 2}{\sqrt{\left(k_{\mathcal{E}_{1}} x_{P_{1}}^{2} / 2\right)^{2}+\left(x_{P_{1}} / 2\right)^{2}}}, \\
\operatorname{sign}\left(x_{P_{1}}\right)=-\operatorname{sign}\left(T_{1} \cdot N_{\mathcal{E}_{1}}\right),
\end{array}\right.
$$

thus:

$$
x_{P_{1}}=\frac{1}{k_{\mathcal{E}_{1}}} \frac{-T_{1} \cdot N_{\mathcal{E}_{1}}}{\sqrt{1-\left(T_{1} \cdot N_{\mathcal{E}_{1}}\right)^{2}}} .
$$

Since $k_{t}=k_{\mathcal{E}_{1}} \cos \beta_{\mathcal{E}_{1}}$, the above expression can be rewritten as:

$$
x_{P_{1}}=\frac{\cos \beta_{\mathcal{E}_{1}}}{k_{t}} \frac{-T_{1} \cdot N_{\mathcal{E}_{1}}}{\sqrt{1-\left(T_{1} \cdot N_{\mathcal{E}_{1}}\right)^{2}}}
$$

$T_{1}, N_{\mathcal{E}_{1}}$ and $\cos \beta_{\mathcal{E}_{1}}$ can be computed from image measurements. Thus, we can determine the distance $\frac{x_{P_{1}}}{2}$ between the intersection of the tangents and $P$ given the curvature $k_{t}$. In addition, it was shown (section 2.2) that the distance between the camera centre $C$ and the intersection of the tangents can also be computed from image measurements. Hence, if this distance is denoted by $d_{1}$, we have the following relation:

$$
\lambda=d_{1}-\frac{1}{2} x_{P_{1}},
$$


which allows the depth at $P$ to be computed given the normal curvature along the viewing direction.

Likewise, since $T$ is also tangent to the second epipolar curve in the epipolar plane $\mathcal{E}_{-1}$, the depth at $P$ can also be computed in the plane $\mathcal{E}_{-1}$. Hence, by (9) and (10):

$$
\left\{\begin{array}{l}
x_{P_{-1}}=\frac{\cos \beta_{\mathcal{E}_{-1}}}{k_{t}} \frac{T_{-1} \cdot N_{\mathcal{E}_{-1}}}{\sqrt{1-\left(T_{-1} \cdot N_{\mathcal{E}_{-1}}\right)^{2}}}, \\
\lambda=d_{-1}-\frac{1}{2} x_{P_{-1}} .
\end{array}\right.
$$

Consequently, we obtain the following system in two unknowns $k_{t}$ and $\lambda$ :

$$
\left\{\begin{array}{l}
\lambda=d_{-1}+\frac{\cos \beta_{\mathcal{E}_{-1}}}{k_{t}} \frac{T_{-1} \cdot N_{\mathcal{E}_{-1}}}{\sqrt{1-\left(T_{-1} \cdot N_{\mathcal{E}_{-1}}\right)^{2}}}, \\
\lambda=d_{1}+\frac{\cos \beta_{\mathcal{E}_{1}}}{k_{t}} \frac{T_{1} \cdot N_{\mathcal{E}_{1}}}{\sqrt{1-\left(T_{1} \cdot N_{\mathcal{E}_{1}}\right)^{2}}} .
\end{array}\right.
$$

Note a crucial property of the above system, i.e., its linearity in $\left(\frac{1}{k_{t}}, \lambda\right)$.

Remark The connection with the depth formula (4) as written in 2.2 becomes clear if we write (see figure 6):

$$
r_{P}-r_{P_{1}}=-\frac{1}{2} x_{P_{1}} T-\sqrt{\left(k_{\mathcal{E}_{1}} x_{P_{1}}^{2} / 2\right)^{2}+\left(x_{P_{1}} / 2\right)^{2}} T_{1}
$$

thus:

$$
\Delta r . N_{1}=-\frac{1}{2} x_{P_{1}} T \cdot N_{1},
$$

and substituting in (4) with $P_{1}=P$ and $P_{2}=P_{1}$ gives:

$$
\lambda=\frac{-\Delta C \cdot N_{1}}{\Delta T \cdot N_{1}}-\frac{1}{2} x_{P_{1}} .
$$

This shows that the second term of (4) is a function of the curvature $k_{t}$ of $\mathcal{S}$ at $P$.

The special case $\cos \beta_{\mathcal{E}_{-1}}=0$ or equivalently $\cos \beta_{\mathcal{E}_{1}}=0$ occurs when the normal $N$ at $P$ is orthogonal to the epipolar plane. This implies that $P$ is a multiple point and thus $\Delta T \cdot N_{ \pm 1}=0$. However, if the camera motion is not along the line of sight at $P(\Delta T \neq 0)$, the depth at such points can still be computed since in this case two or more different image projections of $P$ are available. This points out that $d_{-1}$ and $d_{1}$ should be computed using a robust formula instead of $d_{ \pm 1}=\frac{-\Delta C . N_{ \pm 1}}{\Delta T \cdot N_{ \pm 1}}$ which is not defined at a multiple point (i.e. $\Delta T \cdot N_{ \pm 1}=0$ ). See the appendix for details on how we compute $d_{ \pm 1}$.

Finally, by solving (11) and assuming that camera motions are not along any line of sight, we 
obtain the following solutions:

$$
\left\{\begin{array}{ll}
\lambda=\frac{d_{1} a_{-1}-d_{-1} a_{1}}{a_{-1}-a_{1}}, \\
k_{t}=\frac{a_{1}-a_{-1}}{d_{-1}-d_{1}},
\end{array}\right\} \quad \begin{aligned}
& \text { if } a_{-1} \neq a_{1}, \\
& \lambda=d_{-1}=d_{1},
\end{aligned} \quad \text { if }\left(a_{-1}, a_{1}\right)=(0,0),
$$

where:

$$
\begin{cases}a_{-1}=\cos \beta_{\mathcal{E}_{-1}} \frac{T_{-1} \cdot N_{\mathcal{E}_{-1}}}{\sqrt{1-\left(T_{-1} \cdot N_{\mathcal{E}_{-1}}\right)^{2}}}, & d_{-1}=\frac{-\left(C-C_{-1}\right) \cdot\left(\left(T \wedge T_{-1}\right) \wedge T_{-1}\right)}{\left(T-T_{-1}\right) \cdot\left(\left(T \wedge T_{-1}\right) \wedge T_{-1}\right)}, \\ a_{1}=\cos \beta_{\mathcal{E}_{1}} \frac{T_{1} \cdot N_{\mathcal{E}_{1}}}{\sqrt{1-\left(T_{1} \cdot N_{\left.\mathcal{E}_{1}\right)^{2}}\right.}}, & d_{1}=\frac{-\left(C-C_{1}\right) \cdot\left(\left(T \wedge T_{1}\right) \wedge T_{1}\right)}{\left(T-T_{1}\right) \cdot\left(\left(T \wedge T_{1}\right) \wedge T_{1}\right)}\end{cases}
$$

A geometrical interpretation of $d_{1}$ and $d_{-1}$ is that they represent the distances from the camera centre position $C$ to the viewing line intersections (see 2.2). To give an interpretation of the terms $a_{1}$ and $a_{-1}$, consider the projection of $P_{1}$ and $P_{-1}$ onto the viewing line at $P$. Intuitively, $a_{1}$ and $a_{-1}$ ) can be seen as the positions of these projected points with respect to $P$. Hence, if $P$ is a double point, then either $a_{1}$ or $a_{-1}$ is null and if $P$ is a triple point, then both $a_{1}$ and $a_{-1}$ are null.

Remark The above solutions are not defined if $a_{-1}=a_{1}$ and $\left(a_{-1}, a_{1}\right) \neq(0,0)$. This corresponds to situations where the projections of $P_{-1}$ and $P_{1}$ onto the viewing line at $P$ are the same. Thus, the contributions of the viewing directions $T_{-1}$ and $T_{1}$ in (11) are equal and the system has an infinity of solutions. However, unique solutions for depth and the normal curvature $k_{t}$ at such points $P$ can still be found. The idea is to first compute the depth at one of the epipolar correspondents $P_{1}$ or $P_{-1}$. This can be done by applying (11) at $p_{1}$ (or equivalently $p_{-1}$ ). To this aim, two epipolar correspondents to $p_{1}$ (or equivalently $p_{-1}$ ) must be found. We already have $p$ and we can also use $p_{-1}$ (or equivalently $p_{1}$ ) since $p_{-1}$ and $p_{1}$ are epipolar correspondents in that particular case. Once the depth at $P_{1}$ has been computed, we can determine the depth at $P$ by the fact that they both belong to the same parabola. Then, the equation given by applying (11) at $P$ gives the normal curvature $k_{t} . \triangle$

We conclude that the position of a rim point and the normal curvature $k_{t}$ in the viewing direction can be estimated at any regular point and double point, except if the camera motion is in the viewing direction. For points where more than two rims intersect (i.e., $\left(a_{-1}, a_{1}\right)=(0,0)$ ) and with the same exception, depth can be estimated but not the normal curvature. And if the camera motion is along one of the viewing directions $T_{-1}$ or $T_{1}$, neither curvatures nor the depth at $P$ can be computed. 


\section{Comparison with osculating circle methods}

Most previous works on surface reconstruction from occluding contours [Vai 92, Cip 90, Sze 93, Sea 95, Jos 95] make use of osculating circle methods. In such methods, a rim point $P$ is reconstructed by estimating the osculating circle at $P$ and its epipolar correspondents on the previous and the next rim. To this aim, the viewing lines are projected onto a plane and a circle tangent to these three directions is computed. In [Cip 90, Sze 93, Jos 95] one of the epipolar planes is used and in [Vai 92, Sea 95] the radial plane is used. These approaches implicitly suppose that the camera motion is linear [Cip 90, Sze 93, Jos 95] or that the observed surface is locally cylindrical [Vai 92, Sea 95], and assume that the surface remains on the same side of the tangents in the projection plane.

Our approach avoids such constraint, no assumption has to be made on the camera motion, the local surface shape or the side of the tangents on which the surface lies. In addition, it appears that for non-linear camera motions, part of the rim can not be reconstructed by use of an osculating circle method. See for example figure 7 where the three successive camera positions $C_{-1}, C$, and $C_{1}$ are not aligned.

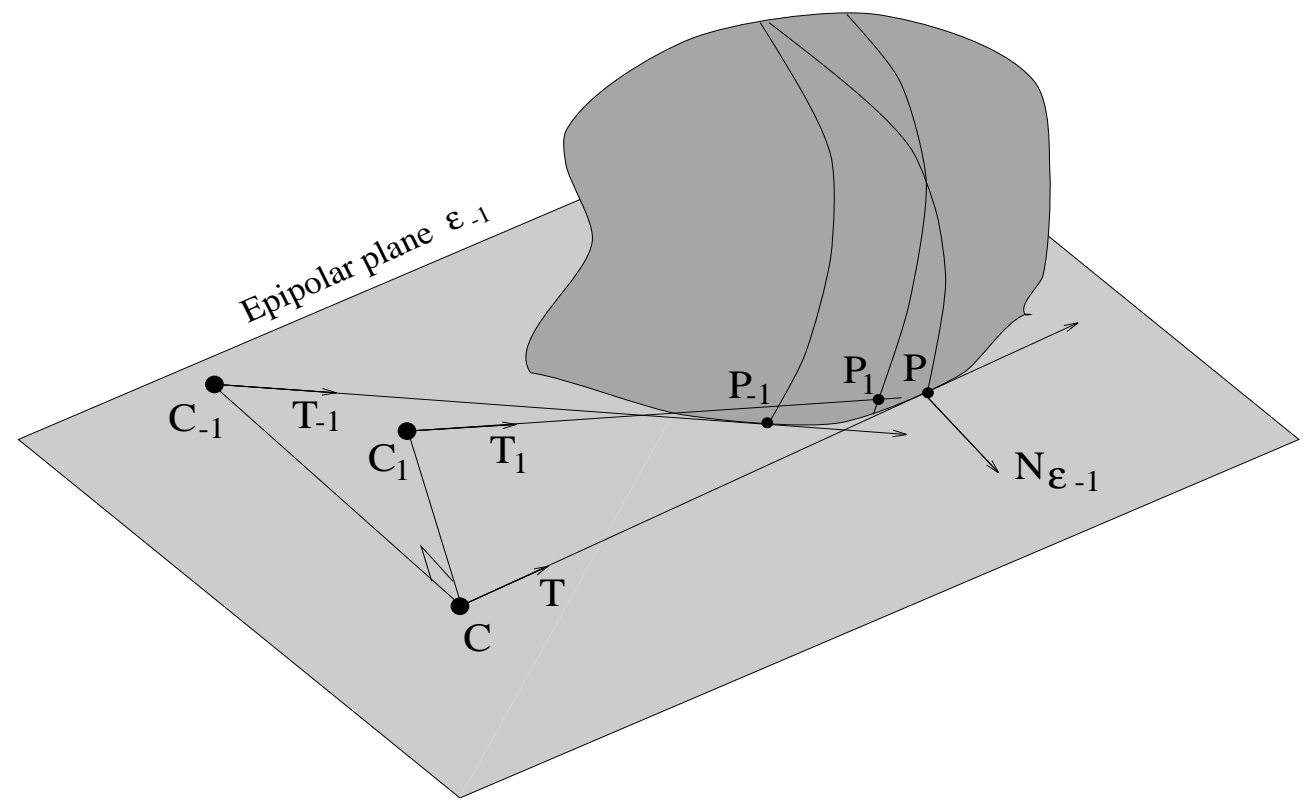

Figure 7: Rims under non-linear camera motion.

In this situation, there is part of the rim to be reconstructed where the point $P$ and its epipolar correspondents are not organised as expected for the estimation of the osculating circle. This corresponds to points $P$ where $a_{1}$ and $a_{-1}$ have the same sign or, in other words, where the projection of $P_{-1}$ and $P_{1}$ onto the viewing line at $P$ are on the same side of $P$. In such cases, the 
epipolar curves defined at $P$ can not be considered as part of the same curve and methods based on the osculating circle lead to false solutions since they approximate both epipolar curves with a single planar curve: a circle (see for example figure 8 ).

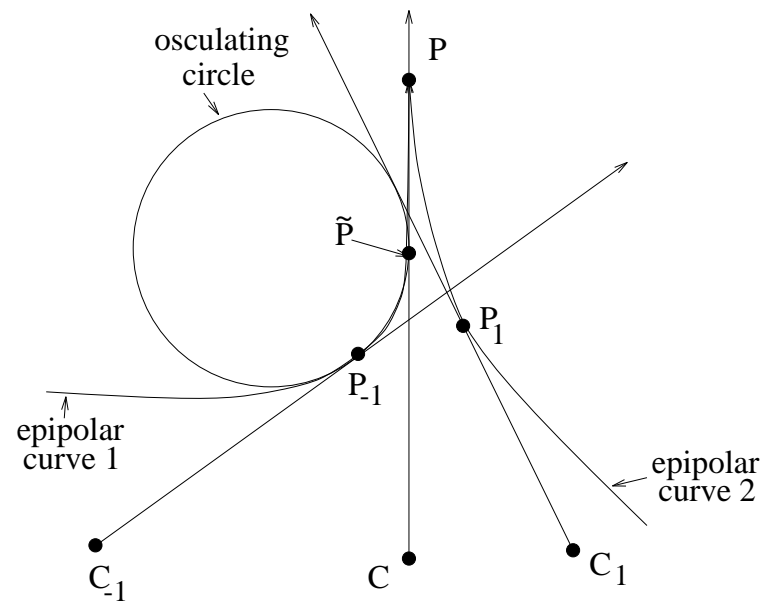

Figure 8: A possible situation for epipolar curves in the case of a non-linear camera motion. The point to be reconstructed is the point $P$ while the position estimated by an osculating circle method is $\tilde{P}$.

This shows that epipolar curves have to be estimated as two different curves and that a method based on the osculating circle allows only a partial reconstruction of the rim in the case of nonlinear camera motions.

Our work gives a more general solution to the reconstruction problem. Except for the special cases where the camera motion is in the viewing direction, depth can be computed at any rim point and for any camera motion. Moreover, it gives a unique solution to the reconstruction problem without knowing on which side of the tangent lines the object lies.

\section{$5 \quad$ Algorithm performances}

In order to determine the accuracy of the reconstruction, we have tested our algorithm on a synthetic sphere. We have chosen synthetic data because it allows computation of exact errors introduced by the reconstruction algorithm. We present here results for plane camera motions.

We suppose that a sphere of radius $200 \mathrm{~mm}$ is observed with a camera positioned $1300 \mathrm{~mm}$ away from the sphere centre. The intrinsic camera parameters correspond to those of a sony ICX camera. A uniform noise is added to the image measurements with a standard deviation of one pixel in both image plane directions. Since a sphere is observed, the normal curvature in the viewing direction $k_{t}$ is equal to the inverse of the sphere radius and the depth is constant for points on one rim. Figure 9 shows the rims observed for plane camera rotations around the sphere centre and figure 10 gives reconstruction errors for two different rotation angles between successive camera positions. 


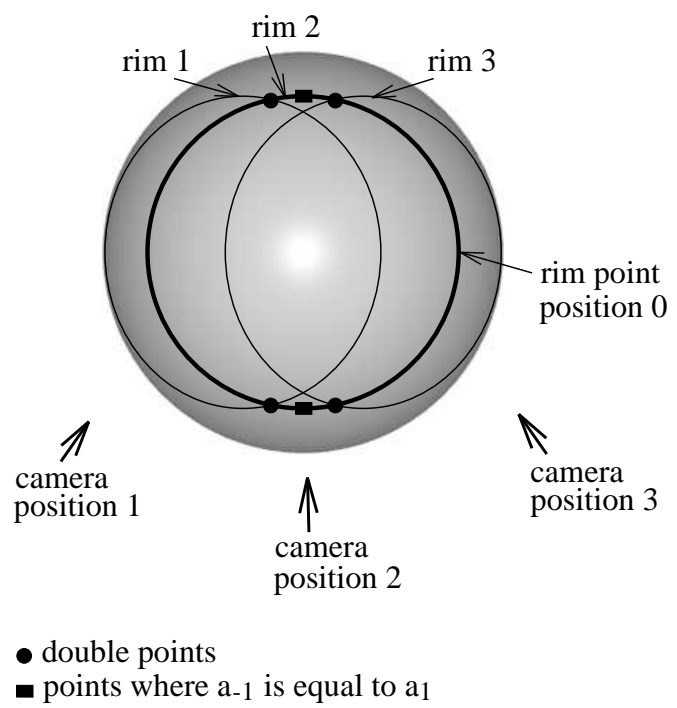

Figure 9: Rims for plane camera rotations around the sphere centre. The rim to reconstruct is $\operatorname{rim} 2$.
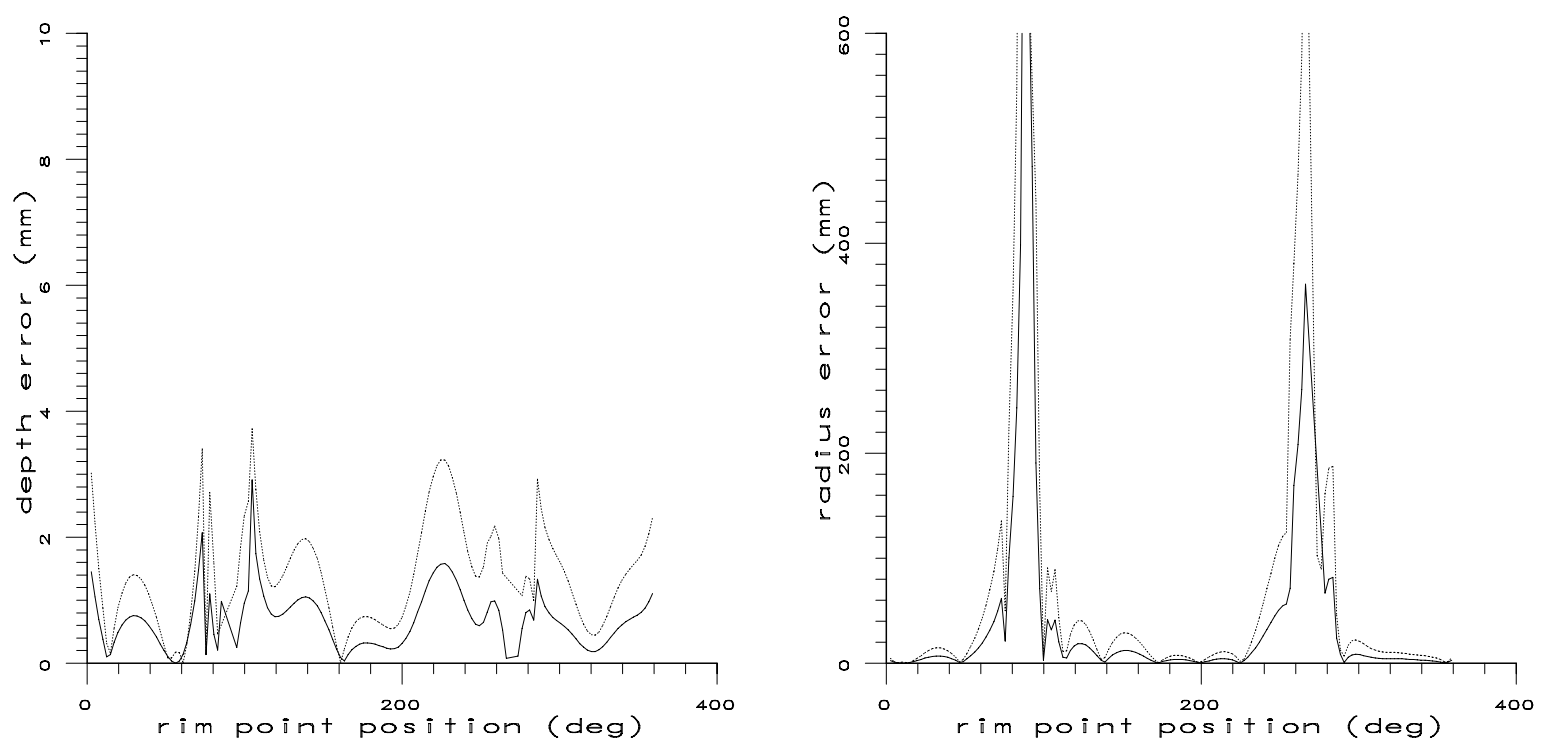

Figure 10: Depth and radius errors for plane camera rotations. The abscissa represents the reconstructed point position on the rim in degree, the position of the origin is shown in figure 9. Solid lines correspond to camera rotations of 10 degrees and dotted lines to camera rotations of 5 degrees. The two peaks in the radius error graph correspond to points where $a_{-1}$ is equal to $a_{1}$.

The depth mean error for a 5 degrees rotation between two camera positions is $1.4 \mathrm{~mm}$ and $0.69 \mathrm{~mm}$ for a 10 degrees rotation. Note that a depth error of $1 \mathrm{~mm}$ yields a distance of $0.0025 \mathrm{~mm}$ between the reconstructed point and the sphere. This shows that a high precision can be expected for rim point positions. On the other hand, the second graph shows that the normal curvature computed is far more sensitive to noise than depth. This is not surprising since depth depends on first order differential property whereas curvatures are second order differential properties of 
the surface. This graph shows also situations where errors increase drastically, namely: points for which $a_{-1}$ and $a_{1}$ are close. In such cases, reconstruction is ill-conditioned. This corresponds, in this example (plane camera rotations), to region of the surface where the three epipolar corresponding points are close. Hence, the estimated curvature is highly biased whereas the depth is still robust. However, for strongly non-linear camera motions, the depth computed is also highly noise sensitive in the vicinity of such points.

In addition, it appears in figure 10 that reconstruction errors increase when the rotation between two successive camera positions decreases. In fact, for a 2 degrees rotation the depth mean error is $3.53 \mathrm{~mm}$ and reaches $9 \mathrm{~mm}$ for a 1 degree rotation. Such noise sensitivity for small camera displacements is due to natural limitations of the reconstruction from images. Indeed, reconstruction algorithms (including stereo-vision) are based on viewing line intersections which are extremely noise sensitive if the viewing directions are close. Hence, very small camera displacements leads to highly biased results and therefore, robust surface reconstruction can not be achieved in such cases.

\section{Experimental results}

We present here results for three real image sequences. Occluding contours were tracked using snakes [Ber 94, Kas 88] (see figures 11(b), 12(b) and 13(b)). The first image sequence consists of 27 images of a tea pot which were taken with unknown camera motions. Therefore a preliminary calibration step is needed. This was done using a calibration pattern which is present in every image (see figure 11(a)). First, discrete points on rims are recovered if they are not close, according to a threshold, to points where $a_{1}$ and $a_{-1}$ are equal. The resulting points are then used to construct a triangular mesh with respect to contour information: two 3D points are connected if and only if they are on two consecutive rims. A ray-tracer is then used to render the surface. Finally, in order to estimate the accuracy of the reconstruction, the rendered surface is projected onto original images. This is done by use of the perspective projection matrix computed during the calibration step. The results of the different steps of the reconstruction process are shown in figure (11).

The reconstructed tea pot shown in this figure is incomplete. This results from the fact that the total camera rotation during the sequence is not sufficient to allow a global perception of the surface. In fact, we reconstruct only what we see and thus a partial surface description may be obtained, depending on the total amount of rotation. This points out that a surface model based on triangular facets is well adapted to modelise the reconstructed surface since it does not require

any a priori information on the surface. Thus, it allows partial as well as complete surface representations without any parametric or topologic information. 
In next examples, real image sequences of a vase and a calabash were taken using a rotating turntable (see figures 12(a) and 13(a)). The rotation angle between two successive images is of 10 degrees for the vase sequence and of 7 degrees for the calabash sequence. Except for the calibration step which was performed before the sequences were taken, the reconstruction process is the same as in the previous example. Results are shown in figures 12 and 13.

Figures 11(f), 12(f) and 13(f) show that reconstructed surfaces are coherent. It should also be noticed that region of the surfaces where $a_{-1}$ is close to $a_{1}$ are still well reconstructed. This is due to the fact that even if these regions correspond to points on one rim for which the reconstruction is ill-conditioned, they may also correspond to points on other rims for which the reconstruction is well-conditioned. Such regions correspond, for the sequences presented, to points which are located at the top and the bottom of the surfaces. Figures 11(c), 12(c) and 13(c) show that the reconstructed rims cover also these regions.

\section{Conclusion}

We have established formulas for the depth and the normal curvature at a point on a rim of a surface. It shows that the computation of the local surface shape can be done with three consecutive occluding contours, even for a non-linear camera motion. Our work extends previous results obtained by others and leads to a general linear solution which is always defined except when the camera motion is in the viewing direction. Moreover, it gives a unique solution to the reconstruction problem without knowing on which side of the tangent lines the object lies. In addition, we have pointed out local properties of the epipolar correspondence and thus situations where this correspondence may fail, namely the multiple points of an image sequence.

This makes it possible to recover the global surface shape. We have tested our algorithm on real data and shown accurate and efficient results on simple objects (i.e., surfaces are at least $C^{2}$ and they are not locally plane). For more complex objects, occluding contours may not be sufficient to allow a complete perception of the object. This is the case, for example, if the object presents concavities. In addition discontinuities may appear on the observed rims and thus a local approximation of the surface by a quadric is not always adapted. See, for example, a composed object such as a coffee cup with a handle; epipolar correspondence may lead to points which belongs to the cup and to the handle, which are two different surfaces. This shows that topological properties of the observed object must be taken into account in the reconstruction process. Our

current work is concerned with rim discontinuities and we plan to integrate different reconstruction methods in order to handle cases such as concavities. 


\section{Appendix}

\section{Computing the distance to the intersection of tangents}

The distance $d_{1}$ from the camera centre position $C$ to the intersection of tangents with direction $T_{1}$ and $T$ verify:

$$
\left(C+d_{1} T-C_{1}-d^{\prime} T_{1}\right)=0, d_{1}=\frac{-\Delta C \cdot N_{1}}{\Delta T \cdot N_{1}} \quad \Delta T \cdot N_{1} \neq 0
$$

where $d^{\prime}$ is the distance from $C_{1}$ to the intersection of tangents with direction $T_{1}$ and $T$. Since $T_{1} \cdot N_{1}=0$ we can therefore write:

$$
d_{1}=\frac{-\Delta C \cdot N_{1}}{\Delta T \cdot N_{1}}, \quad \Delta T \cdot N_{1} \neq 0
$$

However, such a formulation does not hold for a multiple point. Since $d_{1}$ corresponds to the stereo reconstruction of the tangents intersection, it should not depend on the normal $N_{1}$ to the surface. Thus, if we write instead:

$$
d_{1}=\frac{-\Delta C \cdot\left(\left(T \wedge T_{1}\right) \wedge T_{1}\right)}{\Delta T \cdot\left(\left(T \wedge T_{1}\right) \wedge T_{1}\right)}, \quad \Delta T \neq 0
$$

$d_{1}$ is then defined at a multiple point except when the camera motion is along the line of sight $\left(T=T_{1}\right)$. Furthermore, if $T$ and $T_{1}$ are two different viewing directions of a multiple point, then $d_{1}$ is the depth of this point. 


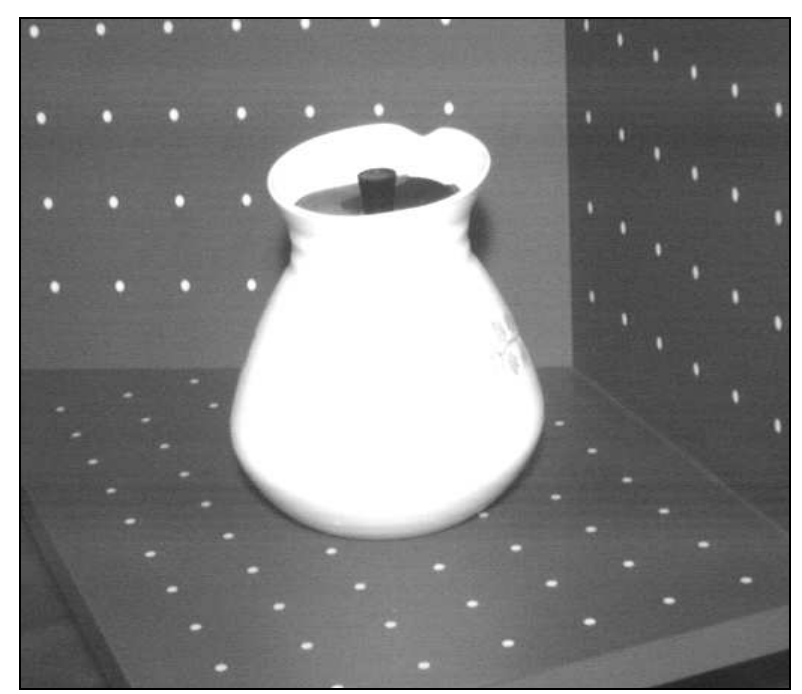

(a)

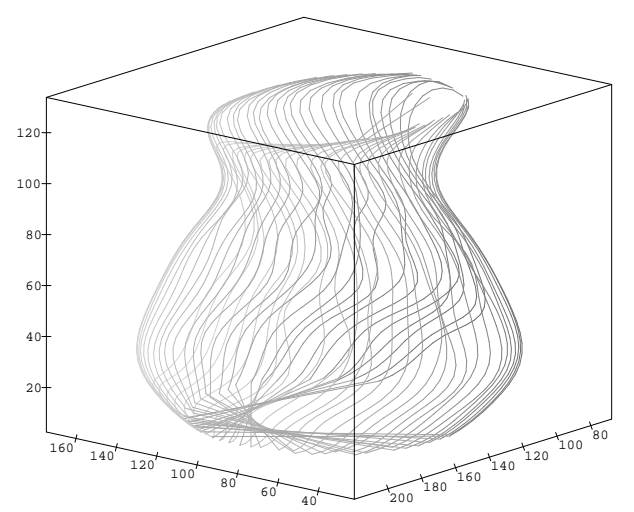

(c)

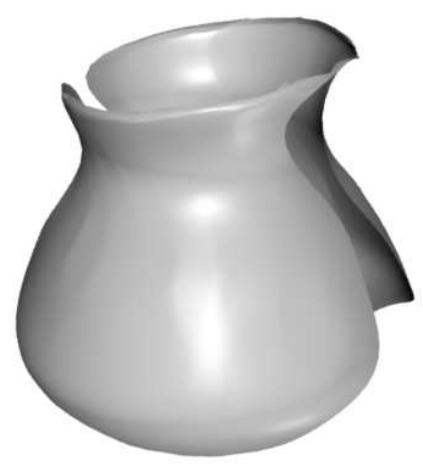

(e)

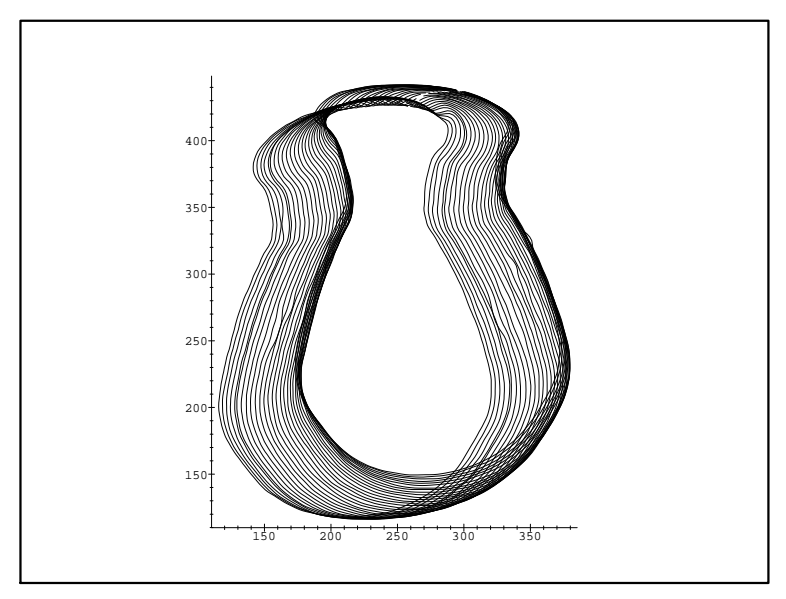

(b)

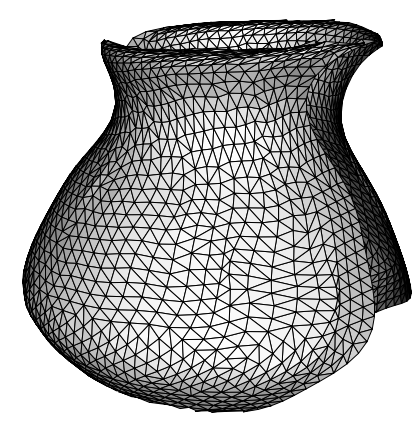

(d)

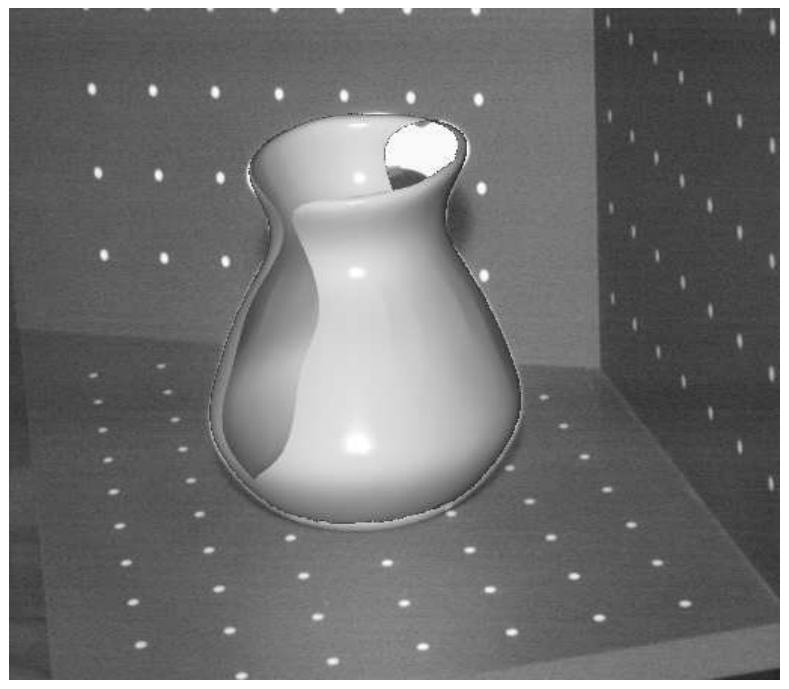

(f)

Figure 11: (a) an image of the sequence, (b) tracked occluding contours, (c) reconstructed rims, (d) triangulated points, (e) rendered surface, (f) projection of the reconstructed surface in the original image. 


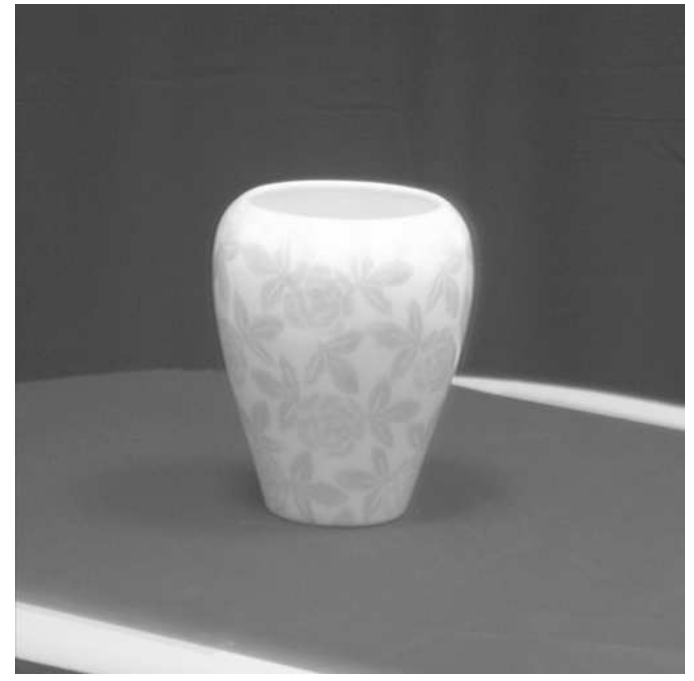

(a)

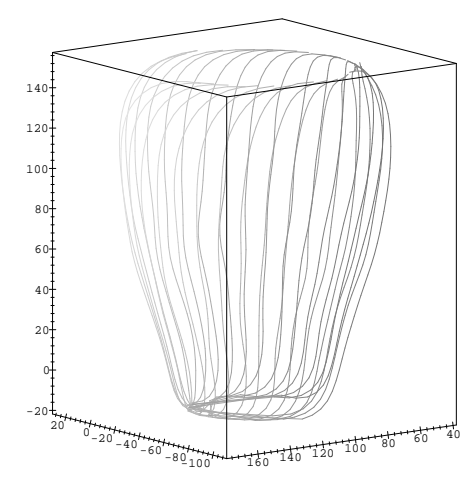

(c)

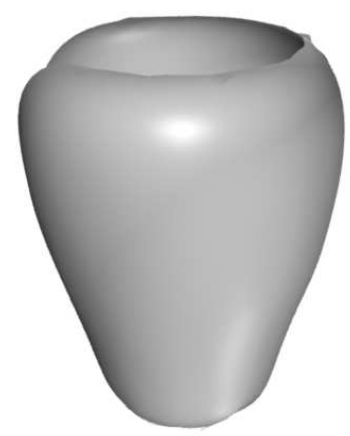

(e)

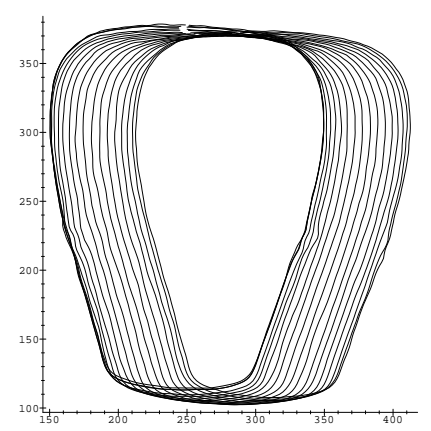

(b)

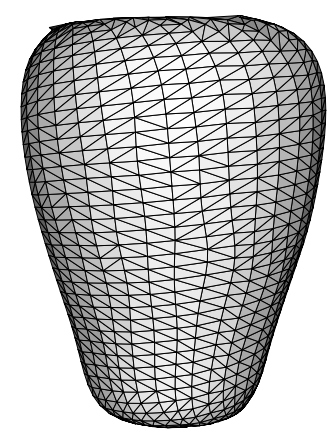

(d)

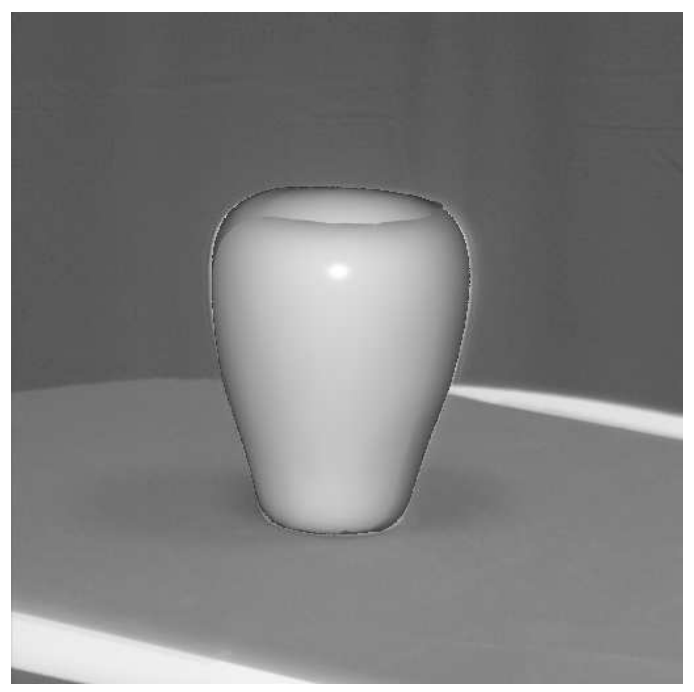

(f)

Figure 12: (a) an image of the sequence, (b) tracked occluding contours, (c) reconstructed rims, (d) triangulated points, (e) rendered surface, (f) projection of the reconstructed surface in the original image. 


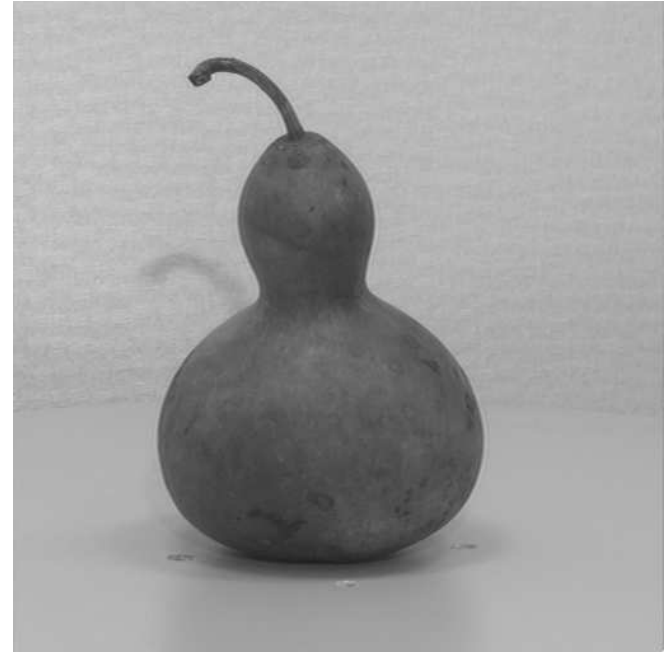

(a)

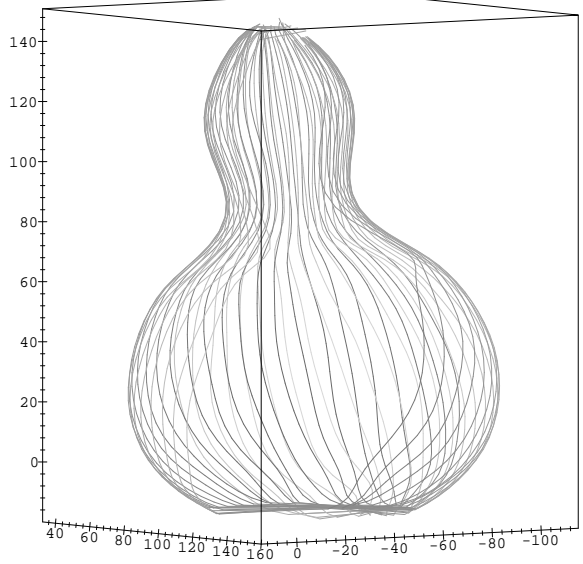

(c)

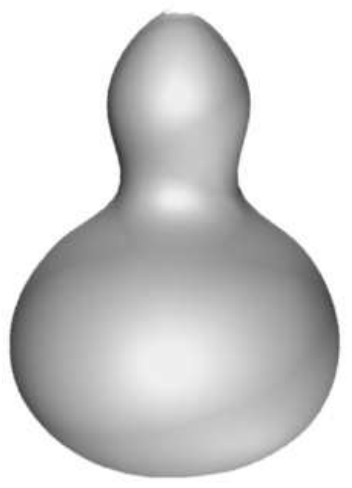

(e)

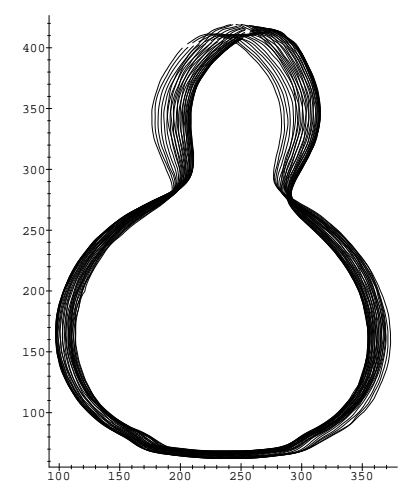

(b)

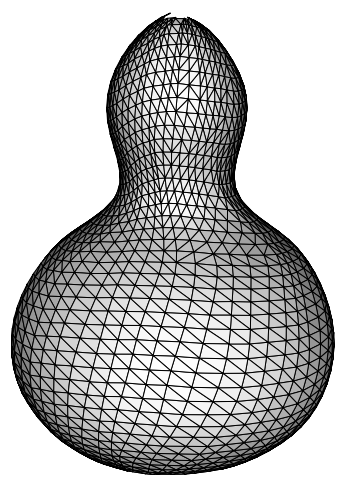

(d)

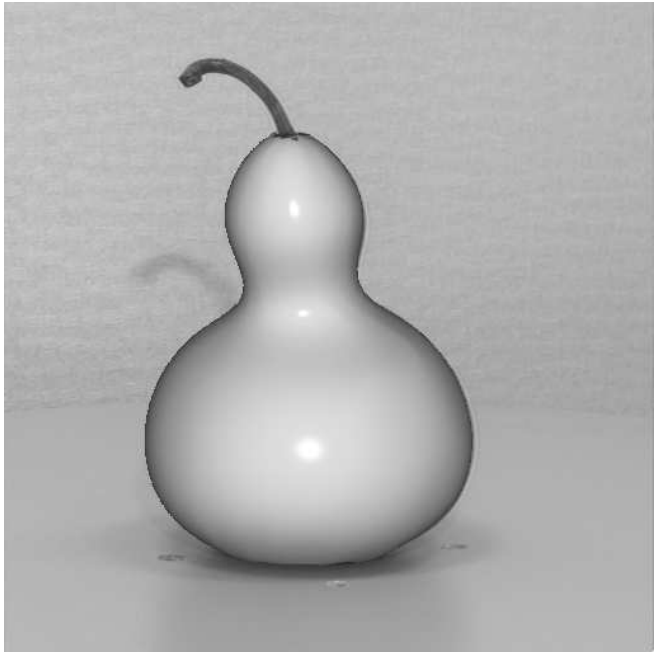

(f)

Figure 13: (a) an image of the sequence, (b) tracked occluding contours, (c) reconstructed rims, (d) triangulated points, (e) rendered surface, (f) projection of the reconstructed surface in the original image. 


\section{References}

[Ber 94] M.-O. Berger. How to Track Efficiently Piecewise Curved Contours with a View to Reconstructing 3D Objects. In Proceedings of the 12th International Conference on Pattern Recognition, Jerusalem (Israel), volume 1, pages 32-36, 1994.

[Bru 84] J.W. Bruce and P.J. Giblin. Curves and Singularities. Cambridge University Press, Cambridge, 1984.

[Cip 90] R. Cipolla and A. Blake. The Dynamic Analysis of Apparent Contours. In IEEE, editor, Proceedings of 3rd International Conference on Computer Vision, Osaka (Japan), pages 616-623, December 1990.

[Cip 95] R. Cipolla, K.E. strm, and P.J. Giblin. Motion from the frontier of curved surfaces. In Proceedings of 5th International Conference on Computer Vision, Boston (USA), June 1995.

[dC 76] M.P. do Carmo. Differential Geometry of Curves and Surfaces. Prentice-Hall, Englewood Cliffs, New Jersey, 1976.

[Fau 93] O. Faugeras. Three-Dimensional Computer Vision: A Geometric Viewpoint. Artificial Intelligence. MIT Press, 1993.

[For 92] D.A. Forsyth, J.L. Mundy, A. Zisserman, and C.A. Rothwell. Recognising Rotationally Symmetric Surfaces from their Outlines. In Proceedings of Second European Conference on Computer Vision, Santa Margherita Ligure (Italy), pages 639-647, May 1992.

[Gib 87] P. Giblin and R. Weiss. Reconstruction of Surfaces from Profiles. In Proceedings of the First International Conference on Computer Vision, London, pages 136-144. IEEE, 1987.

[Gib 95] P.J. Giblin and R.S. Weiss. Epipolar Curves on Surfaces. Image and Vision Computing, 13(1): 33-44, 1995.

[Gla 92] R. Glachet, M. Dhome, and J.T. Lapreste. Finding the Pose of an Object of Revolution. In Proceedings of Second European Conference on Computer Vision, Santa Margherita Ligure (Italy), pages 681-686, May 1992.

[Jos 95] T. Joshi, N. Ahuja, and J. Ponce. Structure and Motion Estimation from Dynamic Silhouettes under Perspective Projection. In Proceedings of 5th International Conference on Computer Vision, Boston (USA), June 1995.

[Kas 88] M. Kass, A. Witkin, and D. Terzopoulos. Snakes: Active Contour Models. International Journal of Computer Vision, 1: 321-331, 1988. 
[Koe 84] J.J. Koenderink. What Does the Occluding Contour Tell us About Solid Shape? Perception, 13: 321-330, 1984.

[Koe 86] J.J. Koenderink. An Internal Representation for Solid Shape Based on the Topological Properties of the Apparent Contour. In W. Richards and S. Ullman, editors, Image Understanding: 1985-86, chapter 9, pages 257-285. Ablex Publishing Corporation, Norwood, NJ, 1986.

[Kri 90] D. Kriegman and J. Ponce. On Recognizing and Positioning Curved 3D objects from Image Contours. IEEE Transactions on PAMI, 12(12): 1127-1137, December 1990.

[Pon 89] J. Ponce, D. Chelberg, and W.B. Mann. Invariant Properties of Straight Homogeneous Generalized Cylinders and their Contours . IEEE Transactions on PAMI, 11(9): 951-966, 1989.

[Por 91] J. Porrill and S. Pollard. Curve matching and stereo calibration. Image and Vision Computing, 9(1): 45-50, 1991.

[Rie 86] J.H. Rieger. Three-dimensional motion from fixed points of a deforming proifle curve. Optics Letters, 11: 123-125, 1986.

[Sea 95] W.B. Seales and O.D. Faugeras. Building Three-Dimensional Object Models From Image Sequences. Computer Vision and Image Understanding, 61(3): 308-324, 1995.

[Sze 93] R. Szeliski and R. Weiss. Robust Shape Recovery from Occluding Contours Using a Linear Smoother. In Proceedings of IEEE Conference on Computer Vision and Pattern Recognition, New York (USA), 1993.

[Vai 92] R. Vaillant and O. Faugeras. Using Extremal Boundaries for 3-D Object Modeling. IEEE Transactions on PAMI, 14(2): 157-173, February 1992.

[Zer 93] M. Zerroug and R. Nevatia. Quasi-Invariant Properties and 3-D Shape Recovery of NonStraight, Non-Constant Generalized Cylinders. In Proceedings of IEEE Conference on Computer Vision and Pattern Recognition, New York (USA), pages 96-103, 1993.

[Zha 94] C. Zhao and R. Mohr. Relative 3D Regularized B-Spline Surface Reconstruction Through Image Sequences. In Proceedings of Third European Conference on Computer Vision (Stockholm, Sweden), volume 2, pages 417-426, May 1994. Lecture Notes in Computer Science, volume 801. 